\title{
Patients Undergoing Bariatric Surgery: a Special Risk Group for Lifestyle, Emotional and Behavioral Adaptations During the COVID-19 Lockdown. Lessons from the First Wave
}

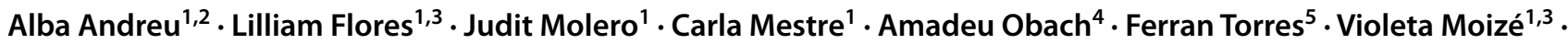 \\ Josep Vidal ${ }^{1,3} \cdot$ Ricard Navinés ${ }^{4} \cdot$ Josep M. Peri ${ }^{4} \cdot$ Silvia Cañizares ${ }^{4,6}$ (i)
}

Received: 2 July 2021 / Revised: 8 November 2021 / Accepted: 10 November 2021 / Published online: 17 November 2021

(c) The Author(s), under exclusive licence to Springer Science+Business Media, LLC, part of Springer Nature 2021

\begin{abstract}
Objectives To determine how the COVID-19 lockdown influenced the lifestyle, eating behavior, use of substances, mental health, and weight in patients who had undergone bariatric surgery (BS) and explore the self-perception of one's own health and fears related to COVID-19.

Methods We performed a cross-sectional exploratory study in obesity patients who had undergone BS surgery $>1$ year previously in a university hospital. Assessment was performed 40 days after initiating lockdown and included 2 periods: from April 24 until May 8 and during the initial de-escalation period: from May 9 until 22, 2020. A structured telephone interview and an online survey were administered.

Results One hundred eighty-eight patients were interviewed; 156 also responded to the online survey (77\% females, mean age $53.46 \pm 10.48$ years, mean follow-up $5.71 \pm 4.30$ years). Dietary habits were affected in $72 \%$ of the participants, with $15 \%$ reporting better diet planning; $83.5 \%$ reported having more sedentary behaviors; $27 \%$ and $36 \%$ showed depression and anxiety, respectively; and $45 \%$ of participants reported bad sleep quality. In relation to changes in the use of any substance, the use increased in the majority of patients who were previously users. Self-perception of one's own health and fears related to COVID-19 were only moderate. Finally, emotional eating and time since BS were statistically significant risk factors for predicting weight gain.

Conclusions Lockdown during COVID-19 pandemic negatively influenced the lifestyle, mental health, substance use, and weight in BS patients. These alterations were somewhat similar to those observed in the general population but more severe and with important clinical implications.
\end{abstract}

Keywords Bariatric surgery $\cdot$ Obesity $\cdot$ COVID-19 $\cdot$ Lockdown $\cdot$ Mental health $\cdot$ Substance use

\section{Key Points}

- The impact of COVID-19 on overall health was more severe in BS patients than in the general population.

- Negative consequences in lifestyle and weight were observed in BS patients during the pandemic.

- BS patients are a group at special risk to the effects of COVID-19 in relation to mental health and substance use.

- Effective therapeutic strategies should be developed for BS during this challenging pandemic.

Alba Andreu and Lilliam Flores contributed equally to this work.

Silvia Cañizares

scanizar@clinic.cat

Extended author information available on the last page of the article

\section{Introduction}

It has been shown that mandatory lockdown periods have a negative psychological impact on the general population. Several emotional disturbances, symptoms of post-traumatic stress, feelings of being emotionally exhausted, reactions of anger, and anxiety, as well as mood alterations and irritability, have been described [1,2]. A study carried out in people in quarantine due to possible contact with patients with severe acute respiratory syndrome (SARS) revealed the presence of emotions of fear, nervousness, sadness, and guilt [3]. In addition, low levels of activity due to confinement also affect night sleep, favoring the appearance of sleep disturbances [4]. 
To detain coronavirus disease 2019 (COVID-19), on March 14, 2020, the Spanish population was ordered to stay home. People were only allowed to leave their homes to purchase essential goods and for going to work in those cases in which in-person work was essential. These strict lockdown indications were extended for 15-day period until May 8, at which time signs of a sustained decline in the infection curve led to progressive de-escalation measures. This deescalation ended in the study area on June 21 after which the area entered a new normality of coexistence with clusters of COVID-19 infection in which face masks and social distancing remained mandatory.

In this context of health crisis, recent literature indicates that patients with obesity have a higher risk of COVID-19 infection and presenting serious and even fatal associated complications [5]. Although some studies have shown a decrease in the prevalence of emotional or psychopathological disorders in subjects with obesity after bariatric surgery (BS), as a group, these individuals continue to present a greater psychopathological burden [6, 7]. Awareness of greater vulnerability in pandemic can increase the level of worry and anxiety. Uncertainty, the duration of confinement, and other variables related to lockdown further affect mood and emotions. These alterations negatively affect eating behavior [8], and can also favor the appearance of emotional eating [9], frequent snacking, and increased alcohol consumption as well as other dysfunctional lifestyle habits, which can lead to overeating and weight regain. Investigation of the aforementioned factors in patients who have undergone $\mathrm{BS}$ is essential to provide preventive public health strategies that can promote the maintenance of the healthiest lifestyles during the pandemic.

Therefore, the primary aim of this study was to determine how the first wave of COVID-19 lockdown influenced the lifestyle, eating behaviors, use of substances, mental health, and weight in patients who had undergone BS. A secondary aim was to explore the self-perception of one's own health and fears related to COVID-19 outbreak.

\section{Materials and Methods}

\section{Design}

We report the cross-sectional part of an ongoing longitudinal study carried out by an Obesity Unit of a university hospital with participants who had undergone BS more than 1 year previously. This exploratory and unicentric study was initially designed to perform two assessments: the first during the lockdown and the second 6 months after the first wave had ended. However, prolongation of the pandemic and the reimposition of the health state of emergency with successive waves of the virus impeded the second assessment in non-confinement conditions at the planned time or thereafter.

The assessment was performed 40 days after having started the lockdown in Spain. Due to changes in confinement measures over time, the assessment was performed into 2 different conditions study periods: one corresponding to the lockdown (from April 24 until May 8) and the other during the initial deescalation period (May 9 until May 22, 2020). The inclusion criteria were primary BS performed more than 1 year previously and scheduled medical or nutritional follow-up visits during the study period. The exclusion criteria were pregnant or breastfeeding patients and patients who had undergone revisional BS. The recruitment was consecutive.

Post-operative follow-up depends on the time since surgery, and as a rule, during the first year after surgery follow-up by endocrinologists and surgeons is performed at 4 and 12 months followed by annually for up to 5 years. Appointments with nutritionists are more frequent during the first two years, being at 15 days, and at 3, 6, 12, 18, 24, and 30 months, followed by annual visits thereafter. Support groups conducted by nutritionists are strongly recommended. When any psychological aspect is detected, presurgery or in the post-surgery follow-up, patients are referred for psychological evaluation and treatment if required.

\section{Methodology}

From the first day of the lockdown, telemedicine practices were implemented. Thus, on the day of the scheduled visit, patients received a telephone instead of face-to-face visit. The aim of the study was explained and informed consent was obtained twice. During the telephone interview, the aim of the study was explained and informed oral consent was obtained. The online survey included the aims of the study, a sentence in which we assured all data would only be used for research purposes, and again, before starting the survey, there was a question about whether the patient wanted to participate. Clicking yes allowed the patient to continue the survey. The study was approved by the Ethical Review Board of our institution (HCB/2020/0567).

The study was carried out in two parts in the same patient: a telephone-interview and an online survey, specifically built by the multidisciplinary members of the Obesity Unit of our hospital. The structured telephone interview collected anthropometric information (subjective change in weight during lockdown) and also some behavioral questions [changes in eating, sleeping and physical activity (PA) habits and changes in substance use (tobacco, cannabis, caffeine, alcohol)]. Furthermore, some validated Spanish questionnaires were administered: a short version of the Alcohol Use Disorders Identification Test, AUDIT-4 [10], taking into account only the period from the beginning of lockdown; the International Physical Activity Questionnaire-Short 
Form (IPAQ-SF) [11, 12] to evaluate PA habits and the Mediterranean Diet Adherence Test (MEDAS) [13]. The latter test was discontinued two weeks after the initiation of the study due to the lengthy time needed to answer the questions and in order to simplify the telephone visits.

The structured online survey included a brief ad-hoc questionnaire oriented to the COVID-19 outbreak (questions about personal and family/relative infection, risk and subjective health perception, etc.), and demographic, work and finance data. In addition, several validated Spanish scales were administered to assess different domains. To evaluate emotional state, we used the Hospital Anxiety and Depression Scale (HADS) [14, 15] and the Positive and Negative Affect Schedule (PANAS) $[16,17]$; three more items were appended reflecting negative affectivity prevalent in the lockdown literature: loneliness, boredom, and frustration. Eating behavior (restrained, external, and emotional) was assessed with the Dutch Eating Behavior Questionnaire (DEBQ) [18, 19]. Sleep quality over a 1-month interval was assessed with the Pittsburgh Sleep Quality Index (PSQI) [20, 21]. Finally, the usual use of alcohol was evaluated with the AUDIT-4 with the instruction to answer according to customary use of alcohol during the previous year (before lockdown). Neuroticism, a personality trait related to health, was assessed with the Neuroticism scale of the Eysenck Personality Questionnaire-Revised (EPQ-R). More information related to the questionnaires and scales used and their scoring is available in Supplementary material 1.

\section{Statistical Analysis}

Continuous variables are described as mean $\pm S D$ values or median [interquartile range (IQR)], according to their adherence to Gaussian distribution. Categorical data are presented as frequencies and percentages. The Fisher's exact, $t$ test, Mann-Whitney, or the Chi-squared test were used when required. To study correlations, we used the Pearson or Spearman coefficients according to the data. Multivariable logistic regression models were used to study predictors of weight gain during the lockdown, considering variables with a $p$ value $\leq 0.10$ on univariate testing for the multivariable analysis using a forward stepwise strategy.

The level of significance was established at the 0.05 level (two-sided) except for the selection of variables for the multivariable logistic model. The statistical analyses were performed using SAS 9.4 (SAS Institute, Inc., Cary, NC, USA).

\section{Results}

\section{Sociodemographic and Clinical Characteristics}

Telephone interviews were performed in 188 patients, 156 of whom also responded to the online survey. Of these, 145 were females (77.1\%). The sociodemographic and clinical characteristics of the sample are shown in Table 1.

There were few differences between the lockdown and deescalation periods. Lesser PA and more sleep disturbances (sleeping $<5 \mathrm{~h}$ ) were reported during lockdown compared to the de-escalation period (PA: $98.2 \%$ vs. $83.3 \%, p=0.0006$; sleep: $14.6 \%$ vs. $3.9 \%, p=0.048)$. Therefore, throughout the manuscript, we will refer to the global sample that comprises the 2 periods except for these 2 variables.

\section{Changes in Lifestyle: Dietary and Physical Activity Habits}

Dietary habits were affected during lockdown in $72 \%$ of the participants, with only $15 \%$ reporting better diet planning. The mean score in the MEDAS $(n=91)$ was $8.54 \pm 2.48$ indicating medium adherence to the Mediterranean diet, and only $25 \%$ of the individuals reported high adherence (Table 2). The MEDAS score only showed an inverse correlation with the body mass index (BMI) $(r=-0.39$ $p<0.001)$.

Regarding PA routines, 173 (92\%) participants reported changes, with 157 (91\%) having more sedentary behaviors (more than $10 \mathrm{~h} /$ day of sitting). According to the IPAQ-SF questionnaire, $69 \%$ of the participants were categorized as having low activity levels (Table 2).

There were no differences according to sex, the type of $\mathrm{BS}$, or the time since surgery in any of the lifestyle measures.

\section{Psychological Variables}

The psychological characteristics of the participants are shown in Table 3. According to HADS, 42 individuals (27\%) suffered some degree of depression and $60 \%$ of them were on antidepressant treatment during the study period. In regard to anxiety, 56 (36\%) of participants presented some degree of anxiety with $55 \%$ receiving psychopharmacological treatment. In relation to sex, the only significant difference was found in anxiety, being more prevalent in women than in men $(40.2 \%$ vs. $21.2 \%$, $p=0.044)$.

Women also exhibited higher negative affectivity compared to men $(22 \pm 8.2$ vs. $17.7 \pm 7, p=0.007)$ in the PANAS. In addition, positive affectivity was directly associated with the MEDAS score $(r=0.334, p=0.002)$ with an inverse correlation coefficient for negative affectivity $(r=-0.217$, $p=0.042$ ). Finally, neither negative emotions related to lockdown (lonely, bored, and frustrated) nor DEBQ scales (emotional, external and restrictive eating) showed differences according to sex, type of BS or time since BS was performed. 
Table 1 Sociodemographic and clinical characteristics of the sample

\begin{tabular}{|c|c|c|c|}
\hline & & Value (mean, $S D$ ) & Range \\
\hline \multirow{4}{*}{$\begin{array}{l}\text { Age (years) } \\
(n=188)\end{array}$} & Total sample & $53.46(10.48)$ & $23-76$ \\
\hline & $\leq 40(n=20,10.6 \%)$ & $34.25(4.45)$ & $23-39$ \\
\hline & $41-59(n=108,57.4 \%)$ & $50.52(5.10)$ & $40-59$ \\
\hline & $\geq 60(n=60,32 \%)$ & $65.17(3.76)$ & $60-76$ \\
\hline \multirow{2}{*}{$\begin{array}{l}\operatorname{Sex}[n(\%)] \\
(n=188)\end{array}$} & Female & $145(77)$ & \\
\hline & Male & $43(22)$ & \\
\hline \multirow[t]{2}{*}{ Lockdown period $[n(\%)](n=188)$} & April 24-May 8 & $109(58)$ & \\
\hline & May 9-May 22 & $79(42)$ & \\
\hline Weight at before lockdown (kg.) $(n=188)$ & & $87.15(18.34)$ & $53-153$ \\
\hline Weight at the survey time $(\mathrm{kg}).(n=188)$ & & $90.04(18.83)$ & $55-157$ \\
\hline BMI at before lockdown $\left(\mathrm{kg} . / \mathrm{m}^{2}\right)(n=188)$ & & $32.08(5.86)$ & $21-56$ \\
\hline BMI at the survey time $\left(\mathrm{kg} . / \mathrm{m}^{2}\right)(n=188)$ & & $32.55(6.01)$ & $17-56$ \\
\hline \multirow{3}{*}{$\begin{array}{l}\text { Type of surgery } \\
{[n(\%)](n=188)}\end{array}$} & Laparoscopic bypass gastric & $87(46)$ & \\
\hline & Laparoscopic sleeve gastrectomy & $90(48)$ & \\
\hline & Duodenal switch & $11(6)$ & \\
\hline \multirow[t]{4}{*}{ Time since BS (years) $(n=188)$} & Total sample (mean, $S D$ ) & $5.71(4.30)$ & $1-15$ \\
\hline & $\leq 2[n(\%)]^{*}$ & $32(17)$ & \\
\hline & $2-5[n(\%)]$ & $62(33)$ & \\
\hline & $>5[n(\%)]$ & $94(50)$ & \\
\hline \multirow{3}{*}{$\begin{array}{l}\text { Level of Education } \\
{[n(\%)](n=156)}\end{array}$} & $\leq$ Undergraduate & $87(56)$ & \\
\hline & Graduate & $24(15)$ & \\
\hline & $\geq$ Postgraduate & $45(29)$ & \\
\hline \multirow{3}{*}{$\begin{array}{l}\text { Employment status } \\
{[n(\%)](n=156)}\end{array}$} & Unemployed & $30(20)$ & \\
\hline & Employed & $88(56)$ & \\
\hline & Retired & $38(24)$ & \\
\hline \multirow[t]{3}{*}{ Marital status [n (\%)] $(n=156)$} & Single & $26(17)$ & \\
\hline & Married/living as couple & $100(64)$ & \\
\hline & Widowed/divorced/separated & 30 (19) & \\
\hline
\end{tabular}

${ }^{*}$ Period $>1$ year but $\leq 2$ years

Table 2 Dietary and physical activity habits during COVID-19 lockdown

\begin{tabular}{lll}
\hline Questionnaire or scale & Mean $(S D)$ \\
\hline MEDAS & Global score (range 0-17) & $8.54(2.48)$ \\
$(n=91)$ & Low adherence $(\leq 7)(\%)$ & 33 \\
& Medium adherence (8-10) $\%)$ & 42 \\
& High adherence $(\geq 11)(\%)$ & 25 \\
IPAQ-SF & Sitting routines (min/day) & $606.23(247.28)$ \\
$(n=188)$ & Walking (METs min/week)* & $331.69(516.83)$ \\
& Moderate intensity (METs min/week) & $100.00(302.53)$ \\
& Vigorous intensity (METs min/week) & $44.47(348.47)$ \\
& Total activity (METs min /week) & $476.15(737.73)$ \\
& Activity Level (\%) & \\
& Low & 69 \\
& Moderate & 29 \\
& High & 2 \\
\hline
\end{tabular}

$M E D A S$, Mediterranean Diet Adherence Test; IPAQ-SF, International Physical Activity Questionnaire-Short Form. *METs, metabolic equivalents
With respect to changes in substance use, tobacco consumption increased in most smokers (84\%). Among participants who changed the use of caffeine, $60 \%$ increased the units/day. The intake of any type of alcoholic beverage changed in only $19 \%$ of the participants, with an increase in alcohol use in $71 \%$ of these individuals. No significant effect was found with regard to time since surgery, marital status, employment status, or worsening of the economic situation due to COVID-19 in any of the changes observed in substance use. The AUDIT-4 corresponding to the lockdown period identified 5\% of risky drinkers, compared to $7.4 \%$ corresponding to the pre-lockdown period. There were no differences between sexes in these percentages but in the global scores, men had higher scores than women in both time periods (lockdown: men mean $=2 \pm 2.4$ vs. women mean $=0.9 \pm 2, p<0.001$; pre-lockdown: men mean $=2.7 \pm 3.83$ vs. women mean $=1 \pm 2.05, p=0.001$ ).

In relation to sleep, the PSQI showed that in the strictest confinement period, more women than men slept $<5 \mathrm{~h}$ $(p=0.039)$. Moreover, women presented worse sleep quality 
Table 3 Phycological, emotional, and mental health factors during COVID-19 lockdown

\begin{tabular}{lll}
\hline Questionnaire or scale & & Mean $(S D)$ \\
\hline HADS & Anxiety & $7.84(3.2)$ \\
$(n=156)$ & Depression & $6.86(3.4)$ \\
& Global score & $14.7(6)$ \\
PANAS (range 5-50) & Positive affect & $29.62(8.2)$ \\
$(n=156)$ & Negative affect & $21.1(8.2)$ \\
Added COVID-19-related & Lonely & $1.92(1.2)$ \\
emotions $*$ (range 1-5) & Bored & $2.51(1.3)$ \\
$(n=156)$ & Frustrated & $2.25(1.3)$ \\
DEBQ & Emotional eating & $2.60(.9)$ \\
$(n=156)$ & External eating & $2.51(.6)$ \\
& Restrained eating & $2.79(.7)$ \\
AUDIT-4 $(n=188)$ & 1 month (during lock- & $1.17(2.2)$ \\
& down) & $1.42(2.5)$ \\
AUDIT-4 $(n=156)$ & 12 months (before lock- & \\
& down) & $43.48(11.3)$ \\
EPQ-R** $(n=156)$ & Neuroticism scale & $7.94(4.3)$ \\
Global PSQI score & & \\
$(n=156)$ & & \\
\hline
\end{tabular}

HADS, Hospital Anxiety and Depression Scale; PANAS, Positive and Negative Affect Schedule; AUDIT-4, Alcohol Use Disorders Identification Test-4 items; $E P Q-R$, Eysenck Personality QuestionnaireRevised; PSQI, Pittsburgh Sleep Quality Index

*Not included in any questionnaire or scale

** Values are expressed in $T$ score (mean 50, DS 10)

\#Significant differences were found according to sex $(p=.021)$

than men $(8.30 \pm 4.45$ vs. mean $6.59 \pm 3.34, p=0.021)$ (Supplementary material 2). In the overall sample, $45 \%$ of participants had bad sleep quality; in $29 \%$, sleep efficiency was $<75 \%, 60 \%$ exhibited sleep latency $>31 \mathrm{~min}$ at least once a week, $72 \%$ reported sleep disturbances, and $29 \%$ used sleep medication.

\section{COVID-19-Oriented Questions}

Regarding self-perception of one's own health, there were no differences according to sex, although there was a significant correlation with years since BS $(r=0.228, p=0.005)$.
Participants demonstrated a greater fear of close family members being infected by COVID-19 and having serious health complications than fear that the same could occur to them (Table 4). In addition, significant, albeit low, correlation coefficients were found between some psychological variables and those fears (range -0.180 to 0.420 ) (Supplementary material 3 ).

Among the survey sample, only $9.8 \%$ had a confirmed nucleic acid-base polymerase chain reaction (PCR) or believed they had had COVID-19 symptoms, and 3 cases required in-patient care. In relation to family members, $11 \%$ had a confirmed PCR or COVID-19 symptoms, with 4 being hospitalized and 3 dying.

\section{Weight Change and Predictors of Subjective Weight Regain}

During lockdown, $72 \%$ of participants believed their weight had changed, and 3\% did not know. Weight gain was the most frequent change reported $(86 \%)$, with a mean of $2.1 \pm 2.8 \mathrm{~kg}$. The weight self-report was significantly greater than the weight recorded before the pandemic $(p<0.001)$ (Table 1).

From among the potential predictors of weight gain found in univariate regression analysis (Supplementary material 4), emotional eating and time since BS were statistically significant risk factors in the multivariable logistic regression model (Table 5), whereas walking METS-min-week showed a non-statistical trend to being a protective factor. The area under the curve was 0.713 .

\section{Discussion}

The results of the present study show that the Spanish COVID-19 lockdown influenced the lifestyle (PA and adherence to the Mediterranean diet), eating behaviors, mental health, substance use, and weight in patients who had undergone BS, a population particularly at risk of adopting maladaptive behaviors in this situation. This is the first study to comprehensively evaluate all these variables in this population.

Table 4 Some of the COVID-19 crisis-oriented questions

\begin{tabular}{ll}
\hline Health and COVID-19-related questions $(n=156)$ & Mean $(S D)$ \\
\hline Self-perception of one's own health (range 1-5) & $3.16(.64)$ \\
Fear of COVID-19 contagion (range 0-10) & $6.09(3.05)$ \\
Fear of getting sick and having serious health complications, and even losing one's life because of COVID-19 (range 0-10) & $5.19(3.32)$ \\
Fear that someone close to you could get COVID-19 (range 0-10) & $7.44(2.75)$ \\
Fear that a close person could become ill and have serious health complications, and even lose their life because of COVID-19 & $7.50(2.81)$ \\
(range $0-10)$ &
\end{tabular}


Table 5 Multivariate logistic regression to predict weight gain during lockdown

\begin{tabular}{|c|c|c|c|c|}
\hline \multirow{3}{*}{$\frac{\text { Predictor }}{\text { Emotional eating (DEBQ) }}$} & \multirow{3}{*}{$\begin{array}{l}p \text { value } \\
.0262\end{array}$} & \multirow{3}{*}{$\frac{O R}{1.52}$} & \multirow{2}{*}{\multicolumn{2}{|c|}{$\begin{array}{l}95 \% \mathrm{CI} \text { for } O R \\
\text { Lower upper }\end{array}$}} \\
\hline & & & & \\
\hline & & & 1.05 & 2.19 \\
\hline Walking METs (100 units) * & .0791 & 0.94 & 0.88 & 1.01 \\
\hline Time since BS & .0219 & 1 & Refe & \\
\hline Time since BS (2-5 yrs.) & .0071 & 4 & 1.46 & 10.99 \\
\hline Time since BS ( $>5$ yrs.) & .0310 & 2.76 & 1.10 & 6.95 \\
\hline
\end{tabular}

$D E B Q$ Dutch Eating Behaviour Questionnaire, *METs metabolic equivalents (IPAQ-SF, International Physical Activity QuestionnaireShort Form)

During lockdown, meal planning worsened in $85 \%$ of the patients studied compared to $36 \%$ in the general Spanish population [22]. This finding is consistent with other studies reporting that following a healthy eating pattern was much more challenging in BS patients [23, 24]. Even though the scores in restrained, emotional and external eating of our participants were analogous to those of the Spanish general population [22], other authors have described an increase in various dysfunctional eating behaviors such as snacking and binge eating [25]. A recent study comparing the evolution of BS patients at 36 months during a non-COVID-19 vs. a COVID-19 period found a significant rise in weight concern, repetitive eating and compulsive grazing in the latter period [26]. Furthermore, inappropriate nutritional intake was found among BS patients in the first year of surgery [27], when individuals are more compliant with dietary recommendations. We found medium adherence to the Mediterranean diet, and the higher the adherence the lower the BMI, similar to findings in the general population [28]. Therefore, the COVID-19 pandemic widely affected different aspects of eating behavior and the following of a healthy diet in BS patients.

It is well known that sedentary behaviors are common among BS candidates and little change in PA have been reported after BS [29]. However, this trend worsened during lockdown in the same line as the general population $(91 \%$ vs. 70\%) [30]. The factors of lifestyle deterioration include not being able to go to work, disruption in routines (family, social, and leisure), fears, and negative emotions [30], particularly in vulnerable groups such as people with obesity or those who have undergone BS [23, 24].

Depression and anxiety symptoms were more prevalent in our group compared with the general Spanish population (27 and $36 \%$ vs. 9 and $8 \%$ respectively) [30], in agreement with the accumulated evidence of a higher prevalence of psychiatric disorders in patients with obesity, even after BS [31], and also with the mental health deterioration described in $\mathrm{BS}$ patients since the beginning of the pandemic [24, 25, 32].

Confinement and social isolation could contribute to the development or worsening of some potentially addictive behaviors, such as online gambling and gaming, as well as the use of any substance as coping strategies [33]. In this regard, we found a significant increase of tobacco, caffeine, and also alcohol consumption similar to other studies [24]. Unexpectedly, risky drinking patterns were slightly lower during lockdown than the year prior to the pandemic (5\% vs. $7 \%$ ), in consonance with data from an international survey [34]. Spending more time with the family and the impossibility of going to establishments that allow consumption could explain these results.

Sleep has frequently been impaired during confinement [24, 30, 35]. Remarkably, $70 \%$ of our global sample presented some kind of sleep disturbances. There is some evidence of an inverse association between sleep quality and $\mathrm{BMI}$ in the general population [35], as well as in individuals who have undergone BS[16]. This relationship cannot be explained only by behavioral components, such as greater eating psychopathology [36]. It has been proposed that short sleep duration may activate obesity-related genes, changes in leptin and ghrelin concentrations and the enhancement of hedonic brain processing that leads to food consumption [37-39].

Strikingly, BS patients exhibited an average self-perception of their own health in contrast to the evidence of a higher risk of being infected and presenting serious health complications [5]. Moreover, self-perception of health was positively influenced by the years since BS was performed. These aspects could, perhaps, reflect a certain degree of health unawareness in the first pandemic wave and the idea that health improves after BS. Fears of COVID-19 contagion and related health complications were only moderate and somehow related to some psychological aspects of negative emotional valence, such as anxiety, negative affectivity, and personality trait neuroticism.

Similar to other studies on patients who have undergone $\mathrm{BS}$, a subjective increase in weight was observed in the vast majority of our patients $(86 \%)$ [40, 41], even from very early on in the pandemic [42]. A study on the 3-year evolution of BS patients during the pandemic compared to prior to the pandemic also showed less weight loss during the pandemic [26]. The changes we observed were nearly twofold greater than those described in the Spanish general population [43]. The weight regain in our study was mild on average $(2.1 \mathrm{~kg})$ but significant in this population. It is likely that the care provided by our team at a medical and nutritional level contributed to mitigating the impact of weight gain during the COVID-19 lockdown.

Among several factors related to surgery, anthropometrics, lifestyle, changes in economic or employment status, 
and psychological variables, only emotional eating and time since BS were risk factors for subjective weight gain. Emotional eating, defined as eating in response to negative emotions or stress, has been described as a way to cope with stressful situations and emotional self-regulation, being highly prevalent in people with obesity. The COVID-19 pandemic has had and have a significant impact on psychological state enhancing negative affectivity [44], and its influence maybe even more prominent in people with obesity as we recently reported [45].

One of the main strengths of this study is the use of validated questionnaires to assess all the domains of interest. When the study was performed, there was little information about the impact of COVID-19 lockdown in BS patients. A limitation of the study is that we only reported crosssectional findings because the second assessment could not be performed as initially designed due to the reimposition of restrictive measures similar to those during the de-escalation period in the first wave of the pandemic. Nonetheless, our findings are relevant to help in reducing the negative impact of the pandemic in this special risk group.

In conclusion, lockdown negatively influenced lifestyle, eating behaviors, mental health, substance use, and weight in BS patients, a special risk group of maladaptive behaviors. These alterations were, to some extent, similar to those reported in the general population but were more severe and had more important clinical implications. These findings will contribute to develop effective therapeutic strategies to address difficulties faced by BS patients, which can be further exacerbated under circumstances such as this challenging pandemic.

Supplementary Information The online version contains supplementary material available at https://doi.org/10.1007/s11695-021-05792-1.

Author Contribution AA, LF, and SC conceived the study, participated in collecting data, contributed to discussion, and wrote the manuscript.

FT performed statistical analysis.

$\mathrm{JM}, \mathrm{CM}, \mathrm{AO}, \mathrm{VM}, \mathrm{RN}, \mathrm{JMP}$, and JV participated in collecting data and contributed to the discussion.

All authors read and approved the final manuscript.

$\mathrm{SC}$ is the guarantor of this work.

Data Availability The datasets generated during and/or analyzed during the current study are available from the corresponding author on reasonable request.

\section{Declarations}

Ethics Approval The study was approved by the Ethical Review Board of our institution (HCB/2020/0567).

Consent to Participate During the telephone visit, we explained the aim of the study and informed oral consent was obtained. Participants were assured all data would only be used for research purposes.
Consent for Publication All participants consented to participate in the study, and accepted the possibility of the publication of a scientific manuscript.

Conflict of Interest The authors declare no competing interests.

\section{References}

1. Brooks SK, et al. The psychological impact of quarantine and how to reduce it: rapid review of the evidence. Lancet. 2020;395(10227):912-20. https://doi.org/10.1016/S01406736(20)30460-8.

2. Cavicchioli M, Ferrucci R, Guidetti M, Canevini MP, Pravettoni $\mathrm{G}$, Galli F. What will be the impact of the covid-19 quarantine on psychological distress? Considerations based on a systematic review of pandemic outbreaks. Healthc. 2021;9(1):101. https:// doi.org/10.3390/healthcare9010101.

3. Reynolds DL, Garay JR, Deamond SL, Moran MK, Gold W, Styra R. Understanding, compliance and psychological impact of the SARS quarantine experience. Epidemiol Infect. 2008;136(7):9971007. https://doi.org/10.1017/S0950268807009156.

4. Altena E, et al. Dealing with sleep problems during home confinement due to the COVID-19 outbreak: practical recommendations from a task force of the European CBT-I Academy. J Sleep Res. 2020;29(4):1-7. https://doi.org/10.1111/jsr.13052.

5. Stefan N, Birkenfeld AL, SchulzeMB. Global pandemics interconnected - obesity, impaired metabolic health and COVID-19. Nat Rev Endocrinol. 2021;17(3):135-149. https://doi.org/10.1038/ s41574-020-00462-1.

6. Livhits $\mathrm{M}$, et al. Behavioral factors associated with successful weight loss after gastric bypass. Am Surg. 2010;76(10):1139-42. https://doi.org/10.1177/000313481007601027.

7. Gill H, Kang S, Lee Y, Rosenblat JD, Brietzke E, Zuckerman H, McIntyre RS. The long-term effect of bariatric surgery on depression and anxiety. J Affect Disord. 2019;1(246):886-894. https:// doi.org/10.1016/j.jad.2018.12.113.

8. Razzoli M, Pearson C, Crow S, Bartolomucci A. Stress, overeating, and obesity: Insights from human studies and preclinical models. Neurosci Biobehav Rev. 2017;76:154-62. https://doi.org/ 10.1016/j.neubiorev.2017.01.026.

9. van Strien T. Causes of emotional eating and matched treatment of obesity. Curr Diab Rep. 2018;18(6):35. https://doi.org/10.1007/ s11892-018-1000-x.

10. Gual A, Segura L, Contel M, Heather N, Colom J. Audit-3 and audit-4: effectiveness of two short forms of the alcohol use disorders identification test. Alcohol Alcohol. 2002;37(6):591-6. https://doi.org/10.1093/alcalc/37.6.591.

11. Rodríguez-Muñoz S, Corella C, Abarca-Sos A, Zaragoza J. Validation of three short physical activity questionnaires with accelerometers among university students in Spain. J Sports Med Phys Fitness. 2017;57(12):1660-8. https://doi.org/10.23736/S00224707.17.06665-8.

12. Craig CL, et al. International physical activity questionnaire: 12-country reliability and validity. Med Sci Sports Exerc. 2003;35(8):1381-95. https://doi.org/10.1249/01.MSS.00000 78924.61453.FB.

13. Álvarez-Álvarez I, et al. Adherence to an energy-restricted mediterranean diet score and prevalence of cardiovascular risk factors in the PREDIMED-plus: a cross-sectional study. Rev Española Cardiol (English Ed). 2019;72(11):925-34. https://doi.org/10. 1016/j.rec.2018.08.010. 
14. Zigmond AS, Snaith RP. The hospital anxiety and depression scale. Acta Psychiatr Scand. 1983;67(6):361. https://doi.org/10. 1111/j.1600-0447.1983.tb09716.x.

15. Herrero MJ, Blanch J, Peri JM, De Pablo J, Pintor L, Bulbena A. A validation study of the hospital anxiety and depression scale (HADS) in a Spanish population. Gen Hosp Psychiatry. 2003;25(4):277-83. https://doi.org/10.1016/S0163-8343(03) 00043-4.

16. Sandín B, Chorot P, Lostao L, Joiner TE, Santed MA, Valiente RM. PANAS positive and negative affect scales: factorial validation and transcultural convergence. Psicothema 1999;11(1):37-51.

17. Watson D, Clark LA, Tellegen A. Development and Validation of Brief Measures of Positive and Negative Affect: The PANAS Scales. J Pers Soc Psychol. 1988;54(6):1063-70. https://doi.org/ 10.1037/0022-3514.54.6.1063.

18. Cebolla A, Barrada JR, van Strien T, Oliver E, Baños R. Validation of the Dutch Eating Behavior Questionnaire (DEBQ) in a sample of Spanish women. Appetite. 2014;73:58-64. https://doi. org/10.1016/j.appet.2013.10.014.

19. van Strien T, Frijters JER, Bergers GPA, Defares PB. The Dutch Eating Behavior Questionnaire (DEBQ) for assessment of restrained, emotional, and external eating behavior. Int $\mathbf{J}$ Eat Disord. 1986;5(2):295-315. https://doi.org/10.1002/1098108X(198602)5:2\%3c295::AID-EAT2260050209\%3e3.0.CO;2-T.

20. Buysse DJ, Reynolds CF, Monk TH, Berman SR, Kupfer DJ. The Pittsburgh sleep quality index: a new instrument for psychiatric practice and research. Psychiatry Res. 1989;28(2):193-213. https://doi.org/10.1016/0165-1781(89)90047-4.

21. Royuela A, Macías JA. Clinimetric properties of the Spanish version of the Pittsburgh questionnaire. Vigilia-Sueño 1997;9(2):81-94.

22. Papandreou C, Arija V, Aretouli E, Tsilidis KK, Bulló M. Comparing eating behaviours, and symptoms of depression and anxiety between Spain and Greece during the COVID-19 outbreak: cross-sectional analysis of two different confinement strategies. Eur Eat Disord Rev. 2020;28(6):836-46. https://doi.org/10.1002/ erv.2772.

23. Almandoz JP, et al. Impact of COVID -19 stay-at-home orders on weight - related behaviours among patients with obesity. Clin Obes. 2020;10(5):1-24. https://doi.org/10.1111/cob.12386.

24. Almandoz JP, et al. Substance use, mental health and weightrelated behaviours during the COVID -19 pandemic in people with obesity. Clin Obes. 2021;11(2):1-10. https://doi.org/10.1111/ cob. 12440 .

25. Athanasiadis DI, et al. "Since January 2020 Elsevier has created a COVID-19 resource centre with free information in English and Mandarin on the novel coronavirus COVID- 19. The COVID-19 resource centre is hosted on Elsevier Connect, the company ' $\mathrm{s}$ public news and information ," no. January, 2020.

26. Conceição E, de Lourdes M, Ramalho S, Félix S, Pinto-Bastos A, Vaz AR. Eating behaviors and weight outcomes in bariatric surgery patients amidst COVID-19. Surg Obes Relat Dis. 2021;17(6):1165-74. https://doi.org/10.1016/j.soard.2021.02.025.

27. Nicoletti CF, et al. Nutritional inadequacies among post-bariatric patients during COVID-19 quarantine in Sao Paulo, Brazil. Obes Surg. 2021;31(5):2330-4. https://doi.org/10.1007/ s11695-020-05107-w.

28. Di Renzo L, et al. Eating habits and lifestyle changes during COVID-19 lockdown: an Italian survey. J Transl Med. 2020;18(1):1-15. https://doi.org/10.1186/s12967-020-02399-5.

29. King WC, et al. Pre- to postoperative changes in physical activity: report from the Longitudinal Assessment of Bariatric Surgery-2 (LABS-2). Surg Obes Relat Dis. 2012;8(5):522-32. https://doi. org/10.1016/j.soard.2011.07.018.

30. Balanzámartínez V, Atienzacarbonell B, Kapczinski F, Deboni RB. Lifestyle behaviours during the COVID-19 - time to connect. Acta Psychiatr Scand. 2020;141(5):399-400.
31. Kalarchian MA, et al. Mental disorders and weight change in a prospective study of bariatric surgery patients: 7 years of followup. Surg Obes Relat Dis. 2019;15(5):739-48. https://doi.org/10. 1016/j.soard.2019.01.008.

32. Sisto A, Vicinanza F, Tuccinardi D, Watanabe M, Gallo IF, D'Alessio R, Manfrini S, Quintiliani L. The psychological impact of COVID-19 pandemic on patients included in a bariatric surgery program. Eat Weight Disord. 2021;26(6):1737-47. https://doi.org/ 10.1007/s40519-020-00988-3.

33. Fernández-Aranda F, et al. COVID Isolation Eating Scale (CIES): analysis of the impact of confinement in eating disorders and obesity - a collaborative international study. Eur Eat Disord Rev. 2020;28(6):871-83. https://doi.org/10.1002/erv.2784.

34. Ammar A, et al. Effects of COVID-19 home confinement on eating behaviour and physical activity : results of the. Nutrients. 2020;12(1583): 13 .

35. Rossi R, et al. COVID-19 pandemic and lockdown measures impact on mental health among the general population in Italy. Front Psychiatry. 2020;11:790. https://doi.org/10.3389/fpsyt.2020. 00790.

36. Brondel L, Romer MA, Nougues PM, Touyarou P, Davenne D. Acute partial sleep deprivation increases food intake in healthy men. Am J Clin Nutr. 2010;91(6):1550-9. https://doi.org/10.3945/ ajen.2009.28523.

37. Chaput JP. "Sleep patterns, diet quality and energy balance,". Physiol Behav. 2014;134, C, 86-91 https://doi.org/10.1016/j. physbeh.2013.09.006.

38. Knutson KL, Spiegel K, Penev P, Van Cauter E. The metabolic consequences of sleep deprivation. Sleep Med Rev. 2007;11(3):163-78. https://doi.org/10.1016/j.smrv.2007.01.002.

39. Valladares M, Obregón AM, Chaput JP. Association between genetic variants of the clock gene and obesity and sleep duration. J Physiol Biochem. 2015;71(4):855-60. https://doi.org/10.1007/ s13105-015-0447-3.

40. de Luis D, et al. Factors related to weight gain in subjects with sleeve gastrectomy during lockdown by the COVID-19 pandemic. Obes Surg. 2021;31(5):2197-202. https://doi.org/10.1007/ s11695-021-05253-9.

41. Félix S, et al. A preliminary study on the psychosocial impact of COVID-19 lockdown in post-bariatric surgery women: the importance of eating behavior, health care access, and social support. Curr Psychol. 2021. https://doi.org/10.1007/s12144-021-01529-6.

42. Walędziak M, et al. Bariatric surgery during COVID-19 pandemic from patients' point of view-the results of a national survey. J Clin Med. 2020;9(6):1697. https://doi.org/10.3390/jcm9061697.

43. López M, Bellido D, Monereo S, Lecube A, Sánchez E, Tinahones FJ. Weight gain during the COVID-19 lockdown ; survey of the Spanish Society of Obesity. Bariátrica \& Metábolica Ibero-Americana. 2020;10.2.4:2774-81.

44. Wang $\mathrm{C}$, et al. "Immediate psychological responses and associated factors during the initial stage of the 2019 coronavirus disease (COVID-19) epidemic among the general population in China,". Int J Environ Res Public Health. 2020;17 5, https://doi.org/10. 3390/ijerph17051729.

45. Jimenez A, et al., "Psychosocial, lifestyle, and body weight impact of COVID-19-related lockdown in a sample of participants with current or past history of obesity in Spain.,”. Obes Surg. 2021, https://doi.org/10.1007/s11695-021-05225-z.

Publisher's Note Springer Nature remains neutral with regard to jurisdictional claims in published maps and institutional affiliations. 


\section{Authors and Affiliations}

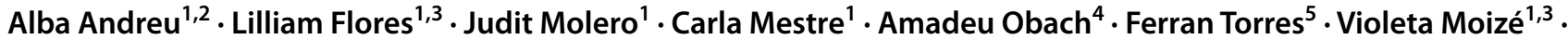 Josep Vidal ${ }^{1,3} \cdot$ Ricard Navinés ${ }^{4} \cdot$ Josep M. Peri ${ }^{4} \cdot$ Silvia Cañizares ${ }^{4,6}$ (i)}

1 Endocrinology and Nutrition Department, Obesity Unit, Hospital Clinic, 170, Villarroel Street, Helios office 9, 08036 Barcelona, Spain

2 CIBER Obesity and Nutrition (CIBEROBN), Barcelona, Spain

3 CIBER Diabetes and Associated Metabolic Diseases (CIBERDEM), Barcelona, Spain
4 Section of Clinical Health Psychology, Psychiatry and Psychology Department, Hospital Clinic, 140 Rosellón Street, 08036 Barcelona, Spain

5 Biostatistics Unit, Faculty of Medicine, Universitat Autònoma de Barcelona; Medical Statistics Core Facility, IDIBAPS, Hospital Clinic Barcelona, Barcelona, Spain

6 Department of Clinical Psychology and Psychobiology, University of Barcelona, Barcelona, Spain 